\title{
Historia ambiental de los archipiélagos de la Trapananda (Patagonia septentrional, Chile): desafíos para la conservación de la ballena azul
}

\author{
Environmental history of the Trapananda Archipelagos \\ (Northern Patagonia, Chile): challenges for the conservation \\ of the blue whale
}

\author{
MARÍA EUGENIA SOLARI \\ Universidad Austral de Chile \\ JUAN CARLOS SKEWES \\ Universidad Alberto Hurtado \\ MAGDALENA NAVARRO \\ Universidad Austral de Chile \\ FABIÁN PAILLACHEO \\ Universidad de Los Lagos
}

RESUMEN La reaparición de la ballena azul en el sur de Chile constituye un desafío para las políticas de conservación e invita a repensar la historia del mar austral y, en particular, de la costa aysenina. El paisaje social de este litoral corresponde a un ecosistema de tránsito y marginalizado entre dos regiones conocidas: Chiloé y Patagonia austral. Es un ambiente extremo en el que diversas poblaciones humanas han desplegado variadas estrategias adaptativas asociadas al bosque y a los sistemas marinos, documentadas en registros arqueológicos, históricos y etnográficos, lo que se procura sintetizar para la reconstrucción de una historia ambiental de la región. Los antece- 
dentes arqueo-históricos dan cuenta de poblaciones canoeras cuya relación con la naturaleza, fundada en un uso extensivo de los recursos, facilitó su integración a los paisajes locales, caracterizados por formaciones archipielágicas. Este patrón se modifica con los procesos históricos desencadenados a partir de la anexión del territorio a los espacios económicos nacionales, situación asociada a sucesivas oleadas poblacionales que se asientan en el litoral de Aysén, modificando las estrategias adaptativas tradicionales. Con la llegada de estas poblaciones se instaura un modelo intensivo y depredador en un paisaje intrínsecamente lábil y, por tanto, agotable. La posibilidad de constituir esta historia en un referente para un adecuado resguardo del área, y, en especial de la ballena azul, especie emblemática cuya presencia se detecta a partir de 2003 , es el desafío a que aquí se alude.

PALABRAS CLAVE Historia ambiental, estrategias adaptativas, ballena azul, litoral de Aysén.

ABSTRACT The reappearance of the blue whale in southern Chile in 2003 has meant a number challenges for national conservation policies. It is also created an opportunity to re-think the history of the southern seas and that of the Aysen coastline in particular. The social landscape of this coastline is characterized by poverty and passage between two well known region Chiloe and Austral Patagonia. This is an extreme environment in which inhabitants have developed a number of adaptive strategies linked to forest and marine systems. These strategies are well documented in the historical, archaeological and ethnographic records which we will gather in order to reconstruct the environmental history of the region. Archaeo-historical records evidenced canoes were vital for the first inhabitants of this area. Their relationship with nature was based on the extensive used of resources which in turn facilitated their integration to the local landscape characterized by the abundance of archipelagos. This relationship was modified after a number of historical processes unchained after the inclusion of this area to the national economy plans. These processes promoted continuous waves of settlement into the region, mainly to the coastline of Aysen, modifying the traditional adaptive strategies and promoting an intensive and abusive used of this intrinsically labile and non-renewable landscape. The opportunity to compile the environmental history of this area and contribute to its protection along with the blue whale is the challenge we present here.

KEYWORDS Environmental history, settlement, blue whale, Aysen's coastline. 


\section{Introducción}

El avistamiento de la ballena azul (Balaenoptera musculus) en el sur de Chile en 2003 plantea un importante desafío: generar políticas e iniciativas de conservación de esta especie, protegida por tratados internacionales, en un contexto de intensa actividad pesquera. La historia ambiental del mar austral sirve de referente para responder a ese desafío. Mediante una perspectiva históricoantropológica y arqueológica de los paisajes litoraleños de la región de Aysén (Patagonia septentional chilena) es posible no sólo reconstruir su historia sino, además, establecer un marco de referencia significativo para enfrentar aquel desafío.

El análisis de la relación entre cultura y ecosistemas acuáticos y terrestres en la región de Aysén, permite efectuar una síntesis que permea las distintas visiones acerca del paisaje holocénico. Este vasto periodo implica revisar variadas adaptaciones a sus ecosistemas terrestres y acuáticos, que incluyen desde la estrategia nómada de los pueblos de cazadores recolectores originarios, hasta las estrategias mixtas de las comunidades actuales, específicamente en el conjunto insular de Guaitecas y Chonos.

En esa línea, uno de los focos de la investigación sociocultural hoy día se centra en procesos y flujos a través de los cuales se movilizan los actores acarreando consigo sus culturas e identidades (Clifford, I997). Por otra parte, la literatura procura dar cuenta de formas de acoplamiento entre las dimensiones humanas y no humanas en la constitución de los paisajes (Ingold, I995; Latour, I999). Es por ello que la mirada antropológica de esta investigación se centra en la interpretación del posicionamiento material e histórico de las poblaciones locales con respecto a los territorios que habitan y sus componentes, como son las comunidades de mamíferos marinos y, más específicamente, especies emblemáticas en ese paisaje, como son los grandes cetáceos.

La historia del complejo territorial y marítimo aysenino puede reconstruirse a partir de la arqueología, los documentos históricos y el trabajo etnográfico con comunidades actuales. Esta historia requiere abordar la relación tierramar como una unidad y el acoplamiento de las poblaciones humanas y no humanas como un todo en el que hay una mutua determinación de las partes. Para aunar estas perspectivas se propone un enfoque derivado de la historia ambiental, tradición que se genera en el campo de la historiografía a partir de los años setenta, momento en el cual la sociedad y la ciencia toman conciencia de la gravedad de la crisis ambiental (González de Molina, I993; Worster, 
2008). Dentro de esta perspectiva, este trabajo permitirá repensar la relación entre seres humanos y naturaleza extrahumana, para reconsiderar los periodos comprendidos por la historiografía solamente y, desde un enfoque interdisciplinario, reconstruir los paisajes arqueológicos e históricos litoraleños de Aysén.

Las aproximaciones metodológicas requeridas para abordar este tipo de procesos de conformación de territorios tienen el carácter de multisituadas (Marcus, 1995; Gudeman y Rivera, I990), toda vez que se hacen cargo de los desplazamientos de los actores, exigiendo el despliegue tanto de la observación directa como de la aplicación de herramientas históricas y analíticas a fin de dar cuenta de los modelos con los que éstos operan (Gudeman, 200I; Wolf, I999).

Una parte importante del poblamiento histórico del territorio aysenino ha sido denominada por la historiografía como colonización particular o espontánea, en contraposición al proceso desarrollado a partir de la entrega por parte del Estado chileno de extensas concesiones de tierra a capitalistas, especuladores particulares y sociedades anónimas que dieron origen a grandes explotaciones pastoriles en la región oriental de la Trapananda, siguiendo el modelo que caracterizaba la actividad empresarial en toda la Patagonia desde fines del siglo XIX (Osorio, 2007). Sin embargo, este proceso de colonización particular o espontáneo es también decidido a partir de la existencia de ciertas materias primas codiciadas, que actualizan el mito de la Ciudad de los Césares, presente en las crónicas, y que incentivan la exploración comercial del territorio.

Los principales procesos migratorios hacia la provincia han tenido como escenario los cursos de aguas marítimas y continentales las que han servido de soporte no sólo a la reproducción de los inmigrados sino que, además, como una vía de transporte. De este modo, el océano se constituye en una gran carretera, espacio de tránsito y contacto, entre poblaciones marinas, como las ballenas, y los pueblos australes (Saavedra, 2007). Los pasos cordilleranos y cursos de agua, a su vez, permiten el desplazamiento de poblaciones terrestres, a las que se suman los cuadros profesionales y colonos movilizados por el Estado en su ejercicio de soberanía geopolítica (figura I).

\section{Arqueología del paisaje aysenino}

El extenso litoral de Aysén estuvo habitado desde el holoceno medio por poblaciones canoeras cuya relación con la naturaleza, fundada en el aprovechamiento extensivo de los recursos, facilitó su permanencia e integración a las 


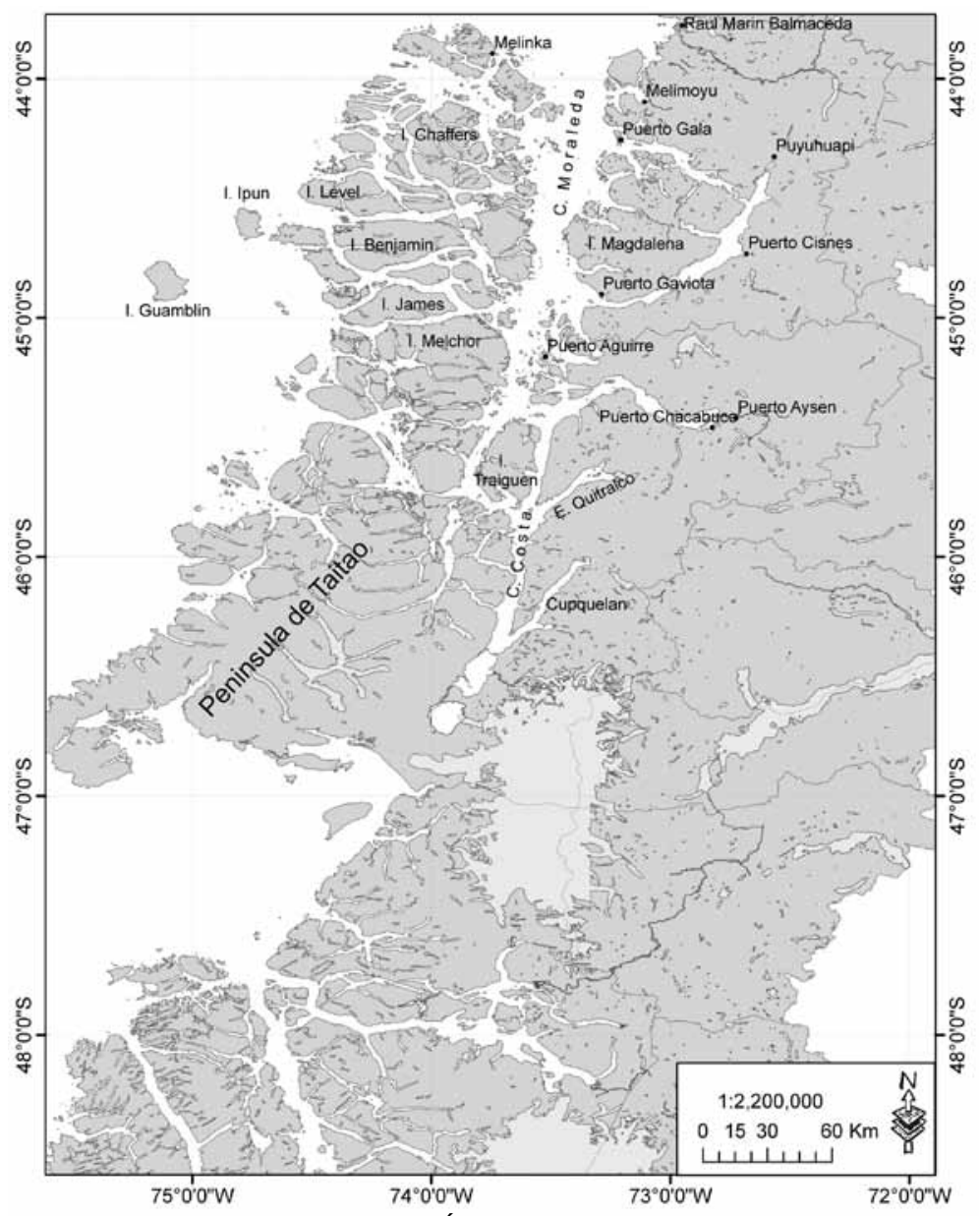

Figura 1. Área de estudio.

formaciones archipielágicas, que caracterizan el ecosistema local (Emperaire, I963; Ocampo y Aspillaga, 2000).

Para Legoupil (2005), en este paisaje de fiordos y archipiélagos dos estrategias son posibles: por una parte una adaptación oportunista al medio ambiente natural y, por otra, un modelo de asentamiento mejor organizado, entre un campo de base o locus preferencial y los sitios periféricos o secundarios. Las poblaciones pescadoras actuales, tanto como las que han sido objeto de la descripción etnográfica tradicional, muestran la interpenetración que se da entre el medio marítimo y el terrestre en que se desenvuelven, poniendo en evidencia la dependencia que tienen del conocimiento del bosque tanto como del mar (Malinowski, 1975; Durrenberger y Palsson, 1987), lo que indudablemente es aplicable a poblaciones arqueológicas. 
La adaptación marítima en los archipiélagos de Aysén, hacia el 5.500 AP, ha sido estudiada de manera fragmentada, en base a la realización de prospecciones arqueológicas parciales. Esta situación dificulta ubicar cronológicamente su posible colonización desde el archipiélago de Chiloé y los intercambios, tanto con los valles orientales, poblados desde el pleistoceno superior, como con las costas y mares interiores de la Patagonia meridional (Emperaire, I963; Ocampo y Aspillaga, I984; Curry, I99 I; Porter, I992; Bird, I993, entre otros); aun así, desde las crónicas y relatos de navegantes, misioneros y etnógrafos, al menos dos pueblos originarios son descritos para este territorio: chonos y kaweshkar. Los primeros habitaron los canales e islas comprendidas entre el archipiélago de las Guaitecas y la península de Taitao. Los kaweshkar, por su parte, conocidos también como alacalufes, poblaron y recorrieron los territorios desde el sur del Golfo de Penas hasta el Estrecho de Magallanes (Emperaire, I963), cuyos descendientes se encuentran asentados principalmente en Puerto Eden. Se trata de territorios permeables que poseían en sus márgenes extensos ecotonos culturales en que las relaciones y dependencias entre los grupos era posible. Los contactos entre estos canoeros australes y quienes habitaban Chiloé debieron darse de manera continua mediante la travesía del Golfo de Corcovado, que delimita a Chiloé del archipiélago de los Chonos. Para los historiadores locales, los espacios litoraleños ayseninos pueden ser vistos como prolongaciones de los espacios nucleares en el mundo chilote.

\section{Los relatos en documentos históricos}

Tempranamente, a mediados del siglo xvi, el mundo archipielágico del litoral aysenino se constituye en lugar de tránsito entre dos espacios conocidos y estratégicos para la corona española: Chiloé y la región de Magallanes (Barros, I98 I; Pastells, I910). A partir de I 553, Francisco de Ulloa y posteriormente el piloto Juan Ladrillero y el marino Francisco Cortés Ojeda describen Guaitecas y Chonos (Pastells, I9ıo; Martinic, 2005). El archipiélago de Chiloé cuenta con fuentes documentales desde el periodo colonial, considerado como un punto crucial para la defensa de la zona austral por la Corona española; asimismo, la Patagonia austral es una de las áreas que presenta la información más temprana. La flota de Magallanes, al cruzar el Estrecho en I 520 , suministra los primeros datos sobre la región (Pigafetta, 1954).

Hacia fines del siglo XVI, las descripciones tanto del litoral de Aysén como de sus habitantes se hacen esporádicas, aluden a un mundo «áspero y montuo- 
so» que no posee gran interés comercial para la corona española. Este desconocimiento permite importar una historia mítica y tejerla para la Trapananda, la de la «Ciudad de los Césares» (Estellé y Couyoudmdjian, I968), que comienza a circular en el siglo xvi bajo la oralidad y la pluma de militares y religiosos (Osorio, 2007: 9). Los viajes misionales del siglo XVII y XVIII se justifican por dos motivos, la cristianización de los indígenas y la búsqueda de esta ciudad presumiblemente enclavada al interior de regiones inexploradas al sur del paralelo 42. (García, I 889; Martinic, 2005). Estas descripciones mostraban una fuerte carga eurocéntrica respecto a la representación del indígena y sus formas de vida, colonización y cristianización.

Paralelamente, desde el siglo XviII hasta inicios del siglo xx, Europa comenzó a interesarse por el conocimiento de la naturaleza sucediéndose las expediciones científicas y de reconocimiento, como las de Moraleda (segundo viaje: I792-I796), Malaspina (I789-I790), Simpson (I872), entre otros (Simpson, I872; Moraleda, I 888; Malaspina, I995).

Desde ese momento, crónicas y relatos varios de viajeros y misioneros que recorrieron el territorio adentrándose para evangelizar o explorar la situación entre los indígenas, tienen a su vez mucho valor para un estudio de la historia del clima y del ambiente (Prieto y otros, s/f). De ellos es posible obtener descripciones como la realizada por el jesuita José de García (I899) quien se aventuró hasta la Laguna San Rafael, en octubre de I766.

Los procesos históricos desencadenados a partir de la anexión del territorio a los espacios económicos nacionales, marca la implantación de modalidades de ocupación alóctona, con la existencia temprana de habitantes provenientes de migraciones cuyos asentamientos, muchas veces efímeros, podrían ser prospectados gracias a la arqueología histórica. Quizás el primer gran hito de «reocupación» estable de estas costas es la fundación de Puerto Melinka en las islas Guaitecas (I 860). Impulsada por la explotación intensiva y extensiva del ciprés de las Guaitecas (Pilgerodendron uviferae), se convierte en la primera de muchas otras permanencias humanas a lo largo del maritorio vinculada a la extracción y manejo de los recursos.

Hacia fines del siglo xIX se produce la conquista del territorio oriental continental y su poblamiento (Riso Patrón, 1905; Steffen, I909), los conflictos de límites políticos con Argentina (Steffen, 1936a, 1936b), la extracción de Ciprés en el litoral (Martinic, 2005), y la búsqueda, esta vez continental, del paso entre Chiloé patagónico y el territorio de Aysén con expedicionarios como Grosse (I986, I990) y Horvath (I978a, I978b, I981). Todas estas acciones 
instauran un modelo intensivo y depredador en un paisaje intrínsecamente lábil y agotable.

El poblamiento «oficial» de las costas de la región de Aysén, relativamente tardío, obedece a variados impulsos de colonización; en una etapa temprana se diferencian claramente dos procesos de asentamiento (Saavedra, 2007):

- la expansión chilota maderera-bentónica;

- la primera colonización impulsada por el Estado.

A principios del siglo xx, las políticas de colonización desde el Estado propician y refuerzan los procesos de poblamiento espontáneos señalados anteriormente, a través de la fundación de Puerto Raúl Marín Balmaceda (I890), Puerto Aguirre (década de I920), Puerto Aysén (I928), Puyuhuapi (I935) y Puerto Cisnes (1954). Estas ubicaciones obedecen a puntos de colonización planificada de acuerdo a las limitaciones o potencialidades en la disponibilidad de materias primas, sumado a la habitabilidad del paisaje.

\section{El escenario actual de las poblaciones litoraleñas de Aysén}

La apertura de Chile a los mercados internacionales, a partir de la década de I980, provoca un auge sin precedentes en la explotación de los recursos del mar, lo que trae consigo un fuerte movimiento de población a lo largo de las costas chilenas. En este contexto se liberan las trabas para la actividad pesquera industrial, facilitando, además, la incorporación de los recursos hidrobiológicos al sistema global de mercado (Duhart, 2004: 5).

En el caso de Aysén, desde I985 en adelante, la explotación de la merluza austral (Merluccius australis), actualiza el repoblamiento litoraleño por inmigrantes, principalmente pescadores y colonos del norte. Paulatinamente, la atracción por la alta demanda del producto y, por ende, su auge económico, da origen a las llamadas «ciudades de plástico», nombre debido al nylon empleado como material de construcción de las barracas que inicialmente albergaron a los primeros habitantes (Hartmann, I995; Rovira, I995).

La apertura a los mercados internacionales causa la gran transformación ocurrida en la costa aisenina ligada a la actividad pesquera y bentónica, en el marco de un territorio que ya era parte del tránsito canoero y puente de los flujos poblacionales que unen el archipiélago de Chiloé y Aysén. Estas migraciones modelan las formas actuales del poblamiento del archipiélago de las Guaitecas, aportando poblaciones desde Chiloé y el sur de Chile, pero también desde Argentina. 
A finales del siglo $\mathrm{xx}$, se gesta, pues, un nuevo fenómeno económico con sensibles implicancias territoriales, sociales y culturales; la expansión de la industria acuícola salmonera como práctica social crea una nueva vida social en el litoral norte aisenino, que desafía y desborda los proyectos de regionalización (Blanco, 2009). Esta actividad se inserta y complementa la vocación económica principalmente bentónica y demersal de estas localidades, amenazando con transformarla en una monoproducción, con altos ingresos económicos.

A partir de 1997, sus fiordos ven multiplicarse las explotaciones salmoneras. La fuerte movilidad asociada al desarrollo de la pesca se intensifica con esta nueva industria, constituyéndose las empresas en un importante atractivo para buzos venidos de las zonas septentrionales del país (Saavedra, 2002, 2007).

\section{Un desafío contemporáneo: la protección de la ballena azul}

Un importante hallazgo en el año 2003 , realizado por un grupo de investigadores, actualiza la valoración de este ecosistema mediante el redescubrimiento científico de agregaciones de mamíferos marinos, específicamente ballenas azules (Balaenoptera musculus) en actividades de alimentación y crianza durante los meses de verano y otoño. La presencia de estas especies emblemáticas que habitan la zona y que han sido objeto de tránsito y confluencia de diversas orientaciones ambientales a lo largo de la historia, plantea nuevos desafíos tanto para las poblaciones litoraleñas como para las políticas asociadas a la protección de esta especie. Desde la perspectiva de la historia ambiental, y más específicamente, desde el modelo propuesto por Worster (2008), podemos aproximarnos a la presencia de estos grupos de mamíferos marinos en la costa de la región de Aysén, patrimonializándolos y convirtiéndolos en un elemento del ecosistema que aglutina recursos disciplinarios desde las ciencias naturales y las ciencias sociales, abriéndose a su vez al ecoturismo, como alternativa económica. Para que ello ocurra se precisa la voluntad de movilizar los recursos locales en función de los intereses de los distintos actores y de sus aliados estratégicos, formular un discurso integrador de las distintas miradas, y ampliar el horizonte cognitivo y emocional acerca del entorno y su relación con la comunidad local (Guerra y Skewes, 2008).

Hoy en día, el área es considerada como la principal zona de alimentación y cuidado parental de éstas y otras especies marinas (Hucke Gaete y otros, 2006). La relación actual de conservación y cuidado de estas especies natura- 
les las ha convertido en elementos claves de resguardo y mantención de estos ecosistemas para los grupos locales.

Las poblaciones de cetáceos han sido fuente de construcciones y representaciones culturales, materiales, económicas e históricas, que se encuentran en directa relación con el sentido de habitabilidad y significación del paisaje costero de Aysén precontacto. Los grupos canoeros del área acceden a los mamíferos marinos a través de actividades de caza o recuperación (carroñeo), que permitían su consumo, fabricación de artefactos y actividades de intercambio (Urbina, I998; Cárdenas, Montiel y Grace, I991).

Por otra parte, el poblamiento histórico alóctono cuantifica este territorio en base a los recursos económicos, incluida la ballena. El dominio de esta esfera por sobre otras dimensiones de interacción con el ambiente natural, gesta, de la mano de inversiones y modelos extranjeros, la introducción en el área de industrias balleneras nacionales, siendo foco de continuos recorridos de barcos balleneros en busca del apetecido producto. Es relevante señalar que dos etapas posee la pesca de ballenas. La primera constituida por exploraciones comerciales de barcos balleneros de bandera extranjera, que operaban en aguas del Pacífico sur oriental desde el Cabo de Hornos hasta las Galápagos (desde I 785 hasta I 880 aprox.). La segunda etapa se desarrolla desde I 880 hasta I98 I aproximadamente, principalmente referida a la captura, faenamiento y comercialización de ballenas por embarcaciones nacionales (Martinic, 2005).

La existencia de las comunidades litoraleñas plantea interrogantes significativas en términos de los patrones que han logrado consolidarse y el impacto que ellos pudieran tener en los ecosistemas locales. La historia ambiental del litoral aisenino revela la fragilidad de un ecosistema que, expuesto a prácticas intensivas de explotación económica, tiende a la destrucción. Es probable que la ballena azul, habiendo recuperado parte de su población, encuentre un freno significativo a su conservación en un contexto donde la liberalización auspicia y estimula acciones desmedidas contra el ambiente. Al examinar la historia ambiental del litoral aisenino se identifican hitos que obran tanto en pro como en contra de su conservación.

La más grave de las amenazas experimentadas por el ecosistema local se refiere a la intensificación de los procesos extractivos y productivos, sea la captura de la merluza austral, sea la proliferación de la salmonicultura, sea la depredación de los recursos bentónicos.

La historia ambiental provee, no obstante, de algunos indicios que bien podrían considerarse para un uso adecuado del litoral, que no exponga la riqueza 
natural a su expoliación por la vía de la faena productiva. Estos hitos están encarnados en la imbricación de las dos zonas aledañas, Chiloé y Patagonia, por la vía de los cursos marinos y continentales que sirven de carretera tanto a las ballenas como a las poblaciones locales. Es este encuentro que merece ser estudiado a cabalidad puesto que, avalado por una vinculación de carácter más bien extensivo con la naturaleza, aseguró la continuidad de las poblaciones humanas y no humanas. Esta clave histórica ambiental invita a conceptualizar y a intervenir en el territorio sobre la base de la conjunción (y no de la disyunción) de los cuerpos de agua y tierra.

El desafío para la conservación supone modificar hábitos de pensamiento y prácticas institucionales que separan agua y tierra. Estas prácticas han favorecido en lo social el desarrollo de poblaciones flotantes, carentes de arraigo territorial. Es preciso recuperar, desde la perspectiva de la planificación, la imagen arqueológica de un territorio permeable que posee en sus márgenes extensos ecotonos culturales que posibilitaban las relaciones y dependencias entre los distintos grupos humanos y especies con las que se relacionan. Es éste el esquema que se fractura con la inmigración desmedida de poblaciones cuyos vínculos se circunscriben a nichos específicos sobre los cuales operan sin consideración al contexto ecológico social. Este desafío requiere, además, recuperar lo que fuera la motivación del siglo XIx: el cultivo del saber científico del territorio aisenino.

\section{Conclusión}

La historia ambiental de la región de Aysén sintetiza, durante los siglos XIX y $\mathrm{xx}$, procesos de ocupación fuertemente vinculados a la presencia de determinados elementos en su ecosistema con alto valor económico, perpetuándose a la vez prácticas culturales ancestrales vinculadas a la caza y recolección en los ecosistemas marino y forestal principalmente. La imagen arqueológica asociada a este periodo es la del entrelazamiento de poblaciones humanas, a través de los cuerpos de agua y de tierra, que permite hacer un uso extensivo no amenazante para las especies con las que aquellas poblaciones se relacionan.

A partir de fines del siglo xix, los paisajes de Aysén viven una especie de re-actualización de la Ciudad de los Césares, al ser entendidos por la población allegada como un territorio mítico vacío y prístino que entrega ganancias económicas, gracias a las riquezas contenidas en su ecosistema natural. La ceguera de este periodo concierne a la negación de una historia humana y am- 
biental en la vastedad austral. Ello permite que la región se repiense con fines mercantiles, en torno al bosque (ciprés principalmente) y el mar, con la caza de la ballena y otros mamíferos marinos, posteriormente por la merluza austral y más recientemente por la industria salmonera.

Estas estrategias actuales sintetizan la movilidad presente en los modelos autóctonos que fluctuaban entre una adaptación oportunista al medio ambiente natural y un modelo organizado en torno a un locus preferencial (Legoupil, 2005), asociado a sitios periféricos o secundarios de extracción de recursos naturales, que es el caso de Melinka, Puerto Aguirre y otras localidades mayores. Al mismo tiempo ponen de relieve el modo como lo local se constituye en torno a procesos de vastos alcances que, en cierto modo, evocan las rutas migratorias de las especies marinas. La irrupción de un modelo alóctono en la ocupación del territorio distorsiona los ejes sobre los que la armazón territorial fue posible; la estrategia oportunista se atrofia de modo que se torna en habitación permante y el episódico y rotativo ingreso de buzos de las costas del sur de Chile a las zonas litoraleñas de Aysén, asociado a las rutas migratorias, deja de ser un medio de aprovisionamiento para convertirse en un medio para lucrar a través de la exportación del producto de la faena.

La revisión de la historia social y ambiental de Aysén entrega claves que permiten pensar de un modo alternativo la relación entre las comunidades humanas, la naturaleza y la ballena azul en tanto especie emblemática de la región. La tarea que sigue consiste en relacionar los procesos migratorios de las poblaciones humanas y sus intersecciones con los avistamientos de esta especie a fin de crear condiciones para una convivencia no destructiva entre ambas poblaciones.

El conocimiento que las ciencias naturales y sociales tienen acerca de los mamíferos marinos y, más específicamente de las ballenas, como elementos claves de resguardo y conservación del ecosistema, y del papel que han jugado en distintos momentos de la historia de estas poblaciones humanas, las convierte en especies de alto interés científico y ecosistémico. Su actual situación, como parte del imaginario social y el reverdecimiento del discurso, permite reconstruir una historia ambiental de estos territorios, al mismo tiempo que vislumbrar una alternativa para que las poblaciones locales puedan ejercer tuición sobre sus medios ambientes, contribuyendo así a la protección de estas especies emblemáticas. 


\section{Referencias}

Barros, J. M. (I98I). Expedición al Estrecho de Magallanes en I 553. Anales Instituto de la Patagonia, I2: 3 I-40.

Bird, J. (I993). Viajes y arqueología en Chile Austral. Punta Arenas: Universidad de Magallanes.

Blanco, G. (2009). The Social Lof Regions. Salmon Farming and the Regionalization of Development in Chilean Patagonia. Tesis de doctorado, Universidad de Wageningen, Holanda.

Cárdenas, R., Montiel, D., y Grace, Y. C. (I99I). Los chonos y los veliche de Chiloé. Santiago: Olimpho.

Clifford, J. (1997). Routes: Travel and Translation in the Late Twentieth Century. Cambridge: Harvard University Press.

Curry, P. (I99I). Distribución de sitios e implicaciones para la movilidad de los canoeros en el canal Messier. Anales Instituto de la Patagonia, 20: I46I 54 .

Duhart, H. (2004). ¿Constituye la legislación actual una herramienta de eficaz para la explotación sustentable del recurso pesquero? Tesis de derecho, Universidad Austral de Chile, Facultad de Ciencias Jurídicas y Administrativas, Valdivia.

Durrenberger, E. P., y Palsson, G. (1987). Ownership at Sea: Fishing Territories and Access to Sea Resources. American Ethnologist, I4 (3): 508-522.

Emperaire, J. (I963). Los nómades del mar. Santiago: U. de Chile.

Estellé, P., y Couyoudmdjian, R. (I968). La ciudad de los Césares: origen y evolución de una leyenda (I 526- I 880). Revista Historia, 7: 283-309.

García, José, S. J. (I 899). Diario del viaje i Navegación hechos por el Padre José García de la Compañía de Jesús desde su misión de Cailin, en Chiloé, hacia el sur, en los años I766 i I767. En AHMCH, año I4, Santiago I 899.

GonZÁlez de Molina, M. (I993). Historia y medio ambiente. Madrid: Eudema.

Grosse, J. (I986). Visión histórica y colonización de la Patagonia occidental. Santiago: Imprenta Mueller.

Grosse, J. A. (1990). Expediciones en la Patagonia occidental. Hacia la carretera austral. Santiago: Andrés Bello.

Gudeman, S. (200I). The Anthropology of Economy. Londres: Blackwell.

Gudeman, S., y Rivera, A. (I990). Conversations in Colombia. The Domestic Economy in Life and Text. Cambridge: Cambridge University Press. 
Guerra D., y Skewes, J. C. (2008). ¿Vernacularización, hibridación, enajenación o patrimonialización? Conserva, I 2: 5-38.

Hartmann, P. (I995). Prehistoria, historia y evolución político-administrativa. Ocupación territorial e interacción entre centros poblados. En Diagnóstico y localización de nuevos centros poblados litoral norte de Aysén. Universidad Austral de Chile y Ministerio de Vivienda y Urbanismo.

Horvath, A. (I978a). Informe preliminar de exploración de alternativa de camino en la región de Aisén. Trapananda, I (I): 2 I-34.

-. (I978b). Informe sobre reconocimiento entre Chile Chico y Mallin Grande por paso de Las Llaves. Trapananda, I (I): 57-60.

-. (I98I). Integración de la zona del Lago General Carrera y el mar. Trapananda, 3 (4): 53-67.

Hucke-Gaete, R., Viddi, F., y Bello, M. (2006). Conservación marina en el sur de Chile. La importancia de la región Chiloé-Corcovado para las ballenas azules, la diversidad biológica y el desarrollo sustentable. Valdivia: Centro Ballena Azul, Universidad Austral de Chile.

INGOLD, T. (I995). Building, dwelling, living. How animals and people make themselves at home in the world. En Marilyn Sthrathern (ed.), Shifting Contexts. Transformations in Anthropological Knowledge. Londres: Routledge. Latour, B. (1999). Pandora's Hope. Essays on the Reality of Science Studies. Cambridge: Harvard University Press.

Legoupil, D. (2005). Rapport de Prospection région d'Aysén. Mission Archéologique Francaise. Manuscrito inédito.

Malaspina, A. (I995). La expedición Malaspina, I789-I794. Diario general del viaje. Madrid: Lunwerg.

Malinowski, B. (I975). Los argonautas del Pacifico Occidental. Barcelona: Península.

Marcus, G. E. (I995). Ethnography In/Of the World System: The Emergence of Multi-sited Ethnography. Annual Review in Anthropology, 24: 95-I I 7.

Martinic, M. (2005). De la Trapananda al Aysén. Santiago: Pehuén.

MoraledA, José de. (I888). Esploraciones jeográficas e hidrográficas practicadas por don José de Moraleda i Montero. Alferez de fragata i primer piloto de la Armada ( $2^{\circ}$ parte correspondiente a los años: I792 a I796). En Анмсн, Santiago, I 888, año XIII.

Ocampo, C., y Aspillaga, E. (I984). Breves notas sobre una prospección arqueológica en los archipiélagos de las Guaitecas y de los Chonos. Revista Chilena de Antropología, 4: I 55-I 56. 
Osorio, M. (2007). Aysén territorio y Aysén humanidad. Itinerario de una construcción social de la(s) identidad(es) regional(es). En M. Osorio, G. Saavedra y H. Velásquez (eds.), Ensayos antropológicos sobre la región de Aysén (9-33). Santiago de Chile.

Pastells, P. (г910). El descubrimiento del Estrecho de Magallanes en conmemoración del IV Centenario del descubrimiento de América. Madrid.

Pigafetta, A (1954). Primer viaje en torno del globo (I 520 ). Buenos Aires: Espasa Calpe.

Porter, C. (I992). GUA-oro, un sitio costero erosionado en una zona sísmica activa. En Actas XII Congreso de Arqueología Chilena. Boletín Museo Regional de Araucanía, 4I: 8I-88.

Prieto, M. R., Solari, M. E., Larraucau, A., y Crouchet, J. (s/f). Fuentes documentales para el conocimiento del clima de los últimos siglos en la región sur de Chile (latitudes 40 a $51^{\circ}$ s.). Manuscrito no publicado.

Riso Patrón, L. (I905). La Cordillera de Los Andes entre las latitudes $46^{\circ} i$ $50^{\circ} \mathrm{S}$. Santiago: Imprenta Cervantes.

Rovira, A. (1995). Ocupación territorial e interacción entre centros poblados. En Diagnóstico y localización de nuevos centros poblados litoral norte de Aysén. Universidad Austral de Chile y Ministerio de Vivienda y Urbanismo.

SaAvedra, G. (2002). Paso al Sur: el litoral norte de Aysén: poblamiento, etnografía y desarrollo. Memoria para optar al titulo de Antropólogo U. de Chile.

-. (2007). Las economías silenciosas del litoral aisenino. En M. Osorio, G. Saavedra y H. Velásquez, Ensayos antropológicos sobre la región de Aysén (35-64). Santiago de Chile.

SAgredo, R., y GonzÁlez, J. I. (2004). La expedición Malaspina en la frontera austral del Imperio español. Santiago: Universitaria.

Simpson, E. (1872). Esploración de las costas occidentales de la Patagonia i del archipiélago de los Chonos, practicada de órden del Supremo Gobierno, en i87 I por el comandante de la corbeta 'Chacabuco' don Enrique M. Simpson. En AUCH, Santiago, I 872, t. XLI, pp. 389-43I.

Steffen, H. (I936a). La cuestión de límites chileno-argentina con especial consideración de la Patagonia. En AUCH, Santiago, I936, pp. I87-23 I.

-. (1936b). Recuerdos del Tribunal Arbitral de Londres. En AUCH, Santiago, I936, pp. 242-256.

-. (1909). Viajes de exploración i estudio en la Patagonia Occidental. Santiago: Imprenta Cervantes. 
Urbina, R. (1988). Los chonos en Chiloé: itinerario y aculturación. Revista de Divulgación del Centro Chilote, 9: 29-42.

Wolf, E. R. (I999). Cognizing 'Cognized Models'. American Anthropologist, IOI (I): I9-22.

Worster, D. (2008). Transformaciones de la Tierra. Montevideo: Editorial Coscoroba y Biblioteca Latinoamericana de Ecología Política de CLAEs.

\section{Sobre los autores}

María Eugenia Solari pertenece al Laboratorio de Arqueobotánica e Historia Ambiental, y al Instituto de Estudios Antropológicos. Es investigadora asociada al Centro de Estudios Ambientales (ceam) de la Universidad Austral de Chile. Su email es <msolari@uach.cl>. Juan CARlos Skewes pertenece al Departamento de Antropología de la Universidad Alberto Hurtado. Es investigador Asociado al Centro de Estudios Ambientales de la Universidad Austral de Chile. Su email es: <jskewes@uahurtado.cl>. Magdalena Navarro pertenece al Equipo Social de la ONG Centro Ballena Azul, Universidad Austral de Chile. Es estudiante del Magíster en Ciencias Sociales, Mención Estudios de Procesos y Desarrollo de las Sociedades Regionales, Centro de Estudios de Desarrollo Local y Regional, Universidad de Los Lagos. Programa Atlas, Universidad de Los Lagos. Tesista Proyecto Fondecyt de Iniciación 11110542. Su correo es <magdalena.nav@gmail.com>. Fabian Paillacheo pertenece al Departamento de Gobierno y Empresa, Universidad de Los Lagos. Su correo es<fabianpaillacheo@hotmail.com>.

Este trabajo está inserto en los proyectos: DID 2008-39 «Historia ambiental y modelos culturales en los ecosistemas marinos de la región de Aysén», perteneciente a la Universidad Austral de Chile (responsable: María Eugenia Solari) y proyecto Fondecyt de Iniciación 11110542 «Escenarios del desarrollo pesquero-artesanal en el contexto de la expansión salmonera. Antropología de las dinámicas económico-culturales en las costas del sur-austral chileno» (investigador responsable: Gonzalo Saavedra Gallo, Universidad Austral de Chile). 